\title{
Vibration-based Health Monitoring of Earth Structures
}

\author{
M. A. Mooney, ${ }^{1, *}$ P. B. Gorman ${ }^{2}$ and J. N. Gonzalez ${ }^{2}$ \\ ${ }^{1}$ Division of Engineering, Colorado School of Mines, Golden, CO, 80401, USA \\ ${ }^{2}$ College of Engineering, University of Oklahoma, Norman, OK, 73019, USA
}

\begin{abstract}
Vibration-based health monitoring (VBHM) has successfully been used to assess the structural damage to bridges, buildings, aircraft, and rotating machinery. There is significant incentive to apply VBHM techniques to the damage detection and conditional assessment of earth structures (geostructures), e.g., foundations, dams, embankments, and tunnels, to improve design, construction, and performance. An experimental program was carried out to explore the efficacy of VBHM of earth structures. A vibratory roller compactor, instrumented with triaxial accelerometers to continuously measure drum and frame vibrations, was operated on a number of underlying material structures with varying properties. Time-domain and frequency-domain analyses of the coupled machine/earth structure response were performed to glean machine vibration features that reflect changes in underlying structural properties. Results illustrate that drum and frame acceleration amplitudes were fairly insensitive to changes in underlying media stiffness; however, drum acceleration frequency components (harmonics) were found to be sensitive to changes in underlying media and changes in soil properties during compaction. The strata underlying the soil undergoing compaction was found to strongly affect drum vibration characteristics.
\end{abstract}

Keywords vibration-based health monitoring - geostructure - soil compaction - vibration techniques · roller compaction · geotechnical

\section{Introduction}

The first civil infrastructure benefactors of structural health monitoring (SHM), and particularly vibration-based health monitoring (VBHM) were bridges and buildings. Earth structures (geostructures) could also benefit from the damage detection and condition assessment capabilities of SHM and VBHM. The makeup of geostructures - including dams, foundations, embankments, roadways - is often complex and uncertain; hence, global/local condition

\footnotetext{
*Author to whom correspondence should be addressed.
}

monitoring and intelligent systems could dramatically improve the design, construction, and operation of such facilities. The construction of geostructures such as earth embankments, foundations, landfills, and highway subgrades is critical to their performance. During earthwork construction, soil is typically compacted using vibration (see Figure 1) to achieve designed-for strength, stiffness, and durability parameters. In current practice, quality assurance and quality control (QAQC) of compaction specifications (e.g., required soil density, moisture content,

Copyright (C) 2005 Sage Publications,

Vol 4(2): 0137-16

[1475-9217 (200506) 4:2;137-16; 10.1177/1475921705049759] 


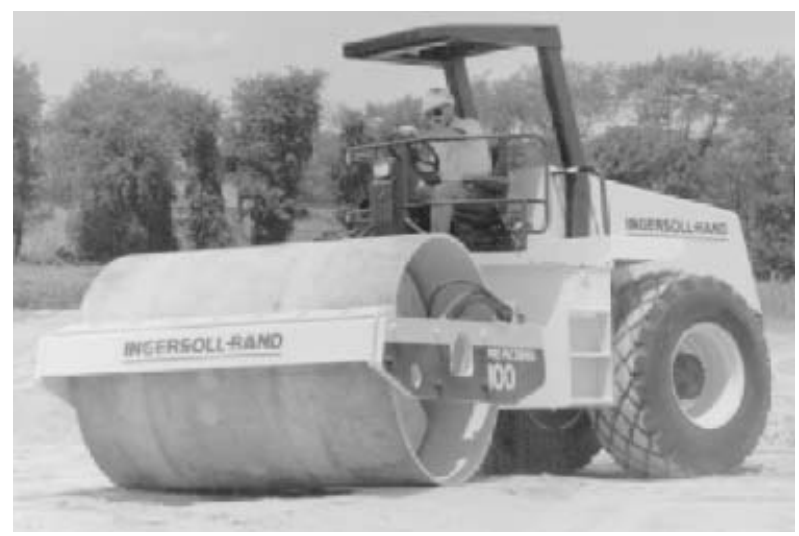

Figure 1 Vibratory roller compactor (courtesy of Ingersoll-Rand Corporation).

resilient modulus) are performed independent of the compaction operation and only at discrete locations. Devices currently employed (and the soil properties measured) include a nuclear gage (moisture content, density), a geogage (low strain stiffness), and a dynamic cone penetrometer (penetration index correlated to shear strength and modulus). Because QAQC operations are performed independent of the compaction operation, the operator is provided with no real-time feedback regarding condition or damage; hence, health monitoring is not being utilized to improve the construction process. Further, because QAQC is performed sporadically at discrete locations, there is no guarantee that the required material properties have been achieved throughout the geostructure.

The construction and performance of geostructures could be greatly enhanced through integration of VBHM into the compaction operation. The effective use of a compactor's vibration characteristics to continuously assess the mechanical properties of the involved soil would constitute a form of continuous quality control and provide a dramatic improvement over current compaction quality control procedures. While onboard 'compaction meters' that monitor drum vibrations are gaining acceptance in practice $[1,2]$, the knowledge base surrounding the relationship between compactor vibration behavior and soil condition is not well-developed. Geostructure
VBHM would be a continuous process throughout construction (i.e., weeks, months), and could be extended to condition/damage assessment throughout the life of the geostructure.

Vibration-based health monitoring techniques have been developed and employed to assess the global condition (health) and detect damage in bridges, buildings, aircraft, and rotating machinery (e.g., see [3]). Changes in material and geometric properties of the system result in measurable changes in vibration characteristics. Both physical model-based and nonphysical model-based approaches have been utilized for system identification of linear and nonlinear systems. The identification of physically or analytically significant features that are derived from response data and are indicators of system condition is critical to the efficacy of health monitoring. Features derived using time-history methods $[4,5]$ and frequency-domain techniques [6] have been successfully used to detect and locate damage.

This paper describes an experimental program undertaken to explore and extract relevant features from the vibratory response of a roller compactor operating continuously within a coupled compactor/geostructure system. While physical model-based nonlinear modeling is under investigation, the immediate goal is to explore a signal-only paradigm (i.e., nonphysical model based) for soil compaction monitoring. Given the precedent set by the rotating machinery industry [7], coupled with the complexity of earthwork compaction, nonphysical model-based condition monitoring is more attractive because it might be more easily implemented in practice. An Ingersoll-Rand Corporation (Shippensburg, PA) smooth drum vibratory roller was instrumented with triaxial accelerometers. Vibration data was first gathered during operation on material structures with significantly different compliances, namely, rubber tires, compacted soil, asphalt pavement, Portland cement concrete (PCC) slabon-grade, and a PCC strong floor. Vibration data were then gathered during the compaction of two geostructures - one made of sand and one of crushed rock. Time and frequency-domain features were determined from the vibration histories, and are presented. 


\section{Experimental Program}

An Ingersoll-Rand SD-100D smooth drum $(2.1 \mathrm{~m}$ wide, $1.5 \mathrm{~m}$ diameter) roller was used during the investigation. The SD-100D has an operating weight of $101 \mathrm{kN}$ and a drum weight of $36 \mathrm{kN}$. The static force measured at the front drum/soil and rear axle/soil contact points are 59 and $42 \mathrm{kN}$, respectively. The vibratory force imparted by the rotating eccentrics within the drum is the vertical component of the centrifugal force:

$$
F_{\mathrm{vib}}=m_{o} e_{o} \varpi^{2} \sin (\varpi t)
$$

where, $m_{o}$ is the mass of the eccentric combination, $e_{o}$ is the eccentricity, and $\varpi$ is the circular frequency of vibration in $\mathrm{rad} / \mathrm{s}$. The SD-100D permits two settings for $e_{o}$ creating $m_{o} e_{o}$ options of $3.1 \mathrm{~kg} \mathrm{~m}$ and $6.2 \mathrm{kgm}$. During low-amplitude vibration on soil $\left(m_{o} e_{o}=3.1 \mathrm{kgm}\right)$, the drum remains coupled with the soil. As the stiffness of the underlying media, vibration frequency, and vibration amplitude increase, the drum can decouple from the soil (bounce). Machine owners and manufacturers prefer to maintain drum/soil coupling to minimize machine wear and tear. Hence, the majority of the test results presented herein involve coupled mode behavior using low vibration amplitude.

The drum and frame of the roller were instrumented with Summit Instruments (Akron,
$\mathrm{OH}$ ) and Crossbow (San Jose, CA) triaxial accelerometers (see Figure 2) aligned to measure vertical and horizontal (two directions) accelerations. The low noise $(5-10 \mathrm{mg} \mathrm{rms})$, high sensitivity $(200-420 \mathrm{mV} / \mathrm{g})$ accelerometers measured drum and frame acceleration within a range of \pm 10 and $\pm 7.5 \mathrm{~g}$, respectively. Acceleration data were sampled at $1 \mathrm{kHz}$ and collected via a 16-bit National Instruments ${ }^{\mathrm{TM}}$ DAQ-card and laptop computer. Both time-domain and frequencydomain analyses were performed on the drum and frame acceleration data to explore the sensitivity of various signal features to the soil compaction process. Method descriptions are embedded within the results sections.

The experimental program was conducted in two phases. Phase One vibration testing was carried out on materials with widely varying stiffness values to create baseline data and to determine the sensitivity of drum and frame vibration characteristics to broad material properties. Table 1 summarizes the different underlying media used in Phase One testing. Note that the properties of Phase One media did not change during vibration, i.e., the compacted subgrade and asphalt properties did not degrade. Also, as denoted by column two in Table 1, the compactor remained stationary during vibration on rubber tires and on the PCC strong floor, and traveled at $0.5 \mathrm{~m} / \mathrm{s}$ during vibration on compacted clay, asphalt, and PCC slab-on-grade. Phase Two testing involved monitoring roller vibration

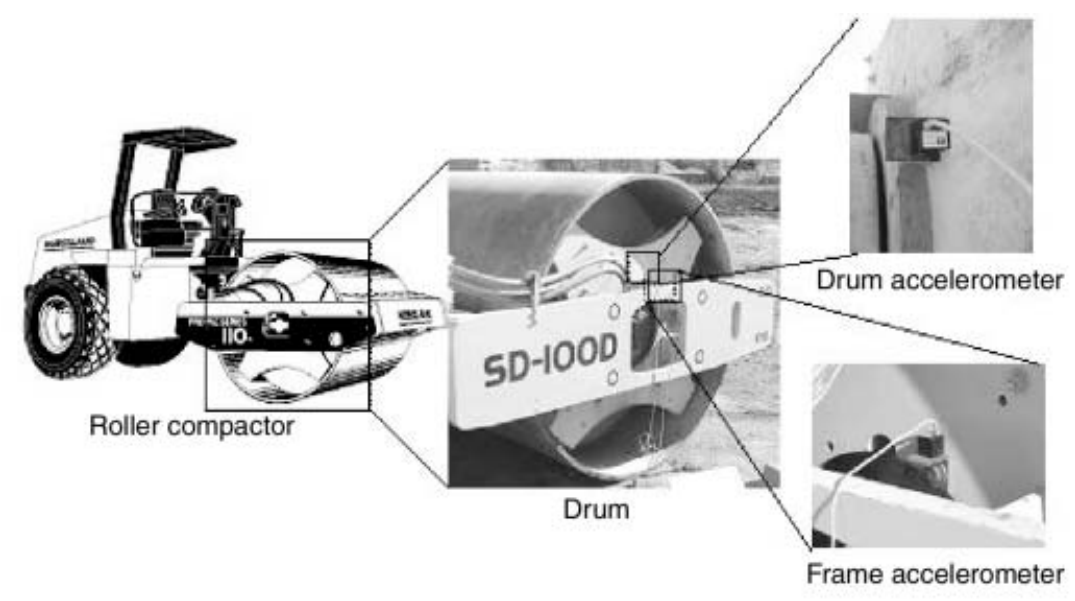

Figure 2 Instrumented roller compactor. 
Table 1 Summary of nonchanging material information.

\begin{tabular}{|c|c|c|c|c|}
\hline Material & Forward velocity & Description & Thickness & Description of subsurface \\
\hline Rubber tires & $0^{\mathrm{a}} \mathrm{m} / \mathrm{s}$ & $\begin{array}{l}\text { Three } 640-\mathrm{mm} \text { diameter, } \\
200-\mathrm{mm} \text { wide rubber } \\
\text { truck tires side by side }\end{array}$ & $200 \mathrm{~mm}$ & $\begin{array}{l}\text { 1-m thick structural concrete } \\
\text { strong floor at the Fears } \\
\text { Structural Engineering } \\
\text { Laboratory }\end{array}$ \\
\hline Compacted clay & $0.5 \mathrm{~m} / \mathrm{s}$ & $\begin{array}{l}\text { Existing compacted low } \\
\text { plasticity clay subgrade }\end{array}$ & $>10 \mathrm{~m}$ & $\begin{array}{l}>10 \mathrm{~m} \text { thick low plasticity dark } \\
\text { brown/red medium clay }\end{array}$ \\
\hline Asphalt & $0.5 \mathrm{~m} / \mathrm{s}$ & $\begin{array}{l}\text { Asphalt concrete road } \\
\text { underlain by aggregate } \\
\text { base and clay subgrade }\end{array}$ & $100 \mathrm{~mm}$ & $\begin{array}{l}>10 \mathrm{~m} \text { thick low plasticity dark } \\
\text { brown/red medium clay }\end{array}$ \\
\hline $\begin{array}{l}\text { Portland } \\
\text { cement } \\
\text { concrete }\end{array}$ & $0.5 \mathrm{~m} / \mathrm{s}$ & $\begin{array}{l}\text { Slab-on-grade foundation } \\
\text { underlain by clay } \\
\text { subgrade }\end{array}$ & $150 \mathrm{~mm}$ & $\begin{array}{l}>10 \mathrm{~m} \text { thick low plasticity dark } \\
\text { brown/red medium clay }\end{array}$ \\
\hline $\begin{array}{l}\text { Portland } \\
\text { cement } \\
\text { concrete }\end{array}$ & $0 \mathrm{~m} / \mathrm{s}$ & $\begin{array}{l}\text { Structural concrete strong } \\
\text { floor at the Fears } \\
\text { Structural Engineering } \\
\text { Laboratory }\end{array}$ & $1 \mathrm{~m}$ & $\begin{array}{l}>10 \mathrm{~m} \text { thick low plasticity dark } \\
\text { brown/red medium clay }\end{array}$ \\
\hline
\end{tabular}

during the compaction of sand and crushed rock test beds. Soil beds, $25 \mathrm{~m}$ (sand) and $10 \mathrm{~m}$ (crushed rock) long and $7 \mathrm{~m}$ wide were carefully prepared with tilling equipment to prepare homogeneous loose soil typical of an earthwork construction environment. The preparation procedure is briefly described below and thoroughly described elsewhere [8].

\section{Phase One Test Results: Vibration on Nonchanging Media}

Drum and frame vibration data were recorded during multiple tests on each of the five media described above and summarized in Table 1. Each test was performed for $60 \mathrm{~s}$ or more; steady-state was achieved within seconds. The drum and soil remained in contact (coupled) during operation on rubber tires, soil, asphalt, and PCC slab-on-grade; however, decoupling occurred during operation on the 1-m thick PCC strong floor. From the time-history acceleration data, peak values of drum and frame accelerations were gleaned for up and down directions and for forward and aft directions. The key steady-state time-domain data summarized in Table 2 for each test includes drum excitation frequency $f_{\mathrm{vib}}$, vibration force $F_{\mathrm{vib}}$ (per Equation (1)), peak values of upward and downward drum accelerations, $\bar{a}_{\mathrm{du}}$ and $\bar{a}_{\mathrm{dd}}$, and peak values of forward and aft drum accelerations, $\bar{a}_{\mathrm{df}}$ and $\bar{a}_{\mathrm{da}}$. Also shown for the drum are the relative difference between upward and downward acceleration peaks $\Delta \bar{a}_{\mathrm{u} / \mathrm{d}}$, the relative difference between forward and aft acceleration peaks $\Delta \bar{a}_{\mathrm{f} / \mathrm{a}}$, as well as normalized vertical and horizontal peak-topeak accelerations $\bar{a}_{z(\mathrm{p}-\mathrm{p})} / F_{\mathrm{vib}}$ and $\bar{a}_{x(\mathrm{p}-\mathrm{p})} / F_{\mathrm{vib}}$. Similar data is presented for frame vibration.

Peak amplitudes of drum and frame acceleration in both the vertical $z$ (upward and downward) and horizontal $x$ (forward and aft) directions are presented together in Figure 3 from machine vibration on rubber tires, compacted soil, asphalt, and PCC slab-on-grade. Positive values indicate upward and forward accelerations while negative values indicate downward and aft accelerations. Each data set presented was gleaned from vibrations at approximately $29 \mathrm{~Hz}$ and each plot is presented at a similar scale to allow visual comparison. The eccentric mass assembly rotates in a forward direction (i.e., with the same trajectory exhibited by the drum rotating as it would move forward); therefore, the $x z$-diagrams are produced by clockwise motion.

Machine vibration on rubber tires was performed to document machine response on a fairly linear elastic underlying medium. Figure 3 illustrates the baseline drum and frame acceleration. Acceleration amplitudes were close to symmetric 
Table 2 Time domain results for vibration on nonchanging materials.

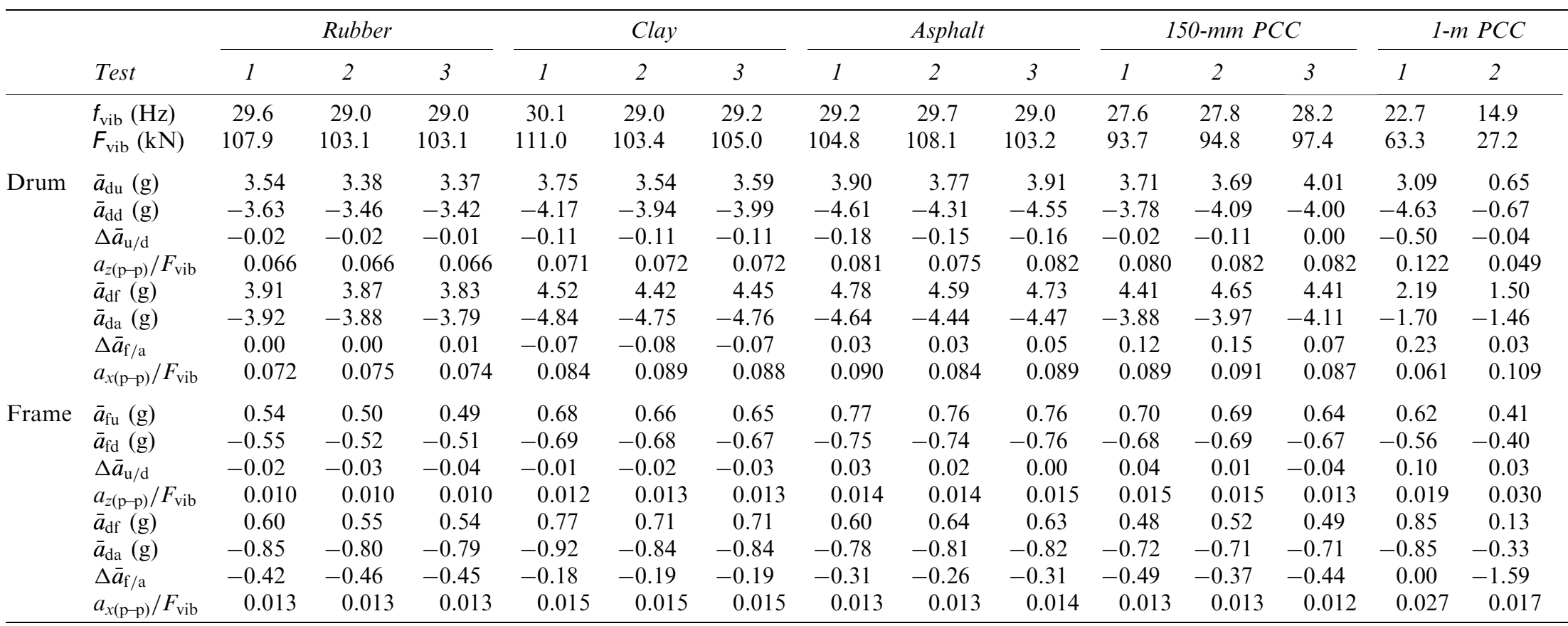

Note: $f_{\text {vib }}$ is drum excitation frequency; $F_{\text {vib }}$ determined from Equation (1); $\bar{a}_{\mathrm{d} / \mathrm{f} u / \mathrm{d}}$ is the mean peak vertical acceleration for the drum (d) and the frame (f) in the upward (u) or downward (d) direction; $\bar{a}_{\mathrm{d} / \mathrm{f} f / a} / 5$ the mean peak horizontal acceleration for the drum (d) and the frame (f) in the forward (f) or aft (a) direction; $\Delta \bar{a}$ is the relative difference in the peak up/forward and peak down/aft acceleration, $\Delta \bar{a}=\left(\left|\bar{a}_{\mathrm{d} / \mathrm{f} \mathrm{u} / \mathrm{f}}\right|-\left|\bar{a}_{\mathrm{d} / \mathrm{f} \mathrm{d} / \mathrm{a}}\right|\right) / \bar{a}_{\mathrm{d} / \mathrm{fu} / \mathrm{f}} \mid$; and $a_{\mathrm{p}-\mathrm{p}} / F_{\mathrm{vib}}$ is the peak to peak acceleration amplitude normalized by generated force with units of $\mathrm{g} / \mathrm{kN}, a_{\mathrm{p}-\mathrm{p}} / F_{\mathrm{vib}}=\left(\left|\bar{a}_{\mathrm{d} / \mathrm{f}} \mathrm{u} / \mathrm{f}\right|+\left|\bar{a}_{\mathrm{d} / \mathrm{f} \mathrm{d} / \mathrm{a}}\right|\right) / F_{\mathrm{vib}}$, where $a_{z(\mathrm{p}-\mathrm{p})} / F_{\mathrm{vib}}$ is the vertical value and $a_{x(\mathrm{p} p)} / F_{\text {vib }}$ is the horizontal value. 

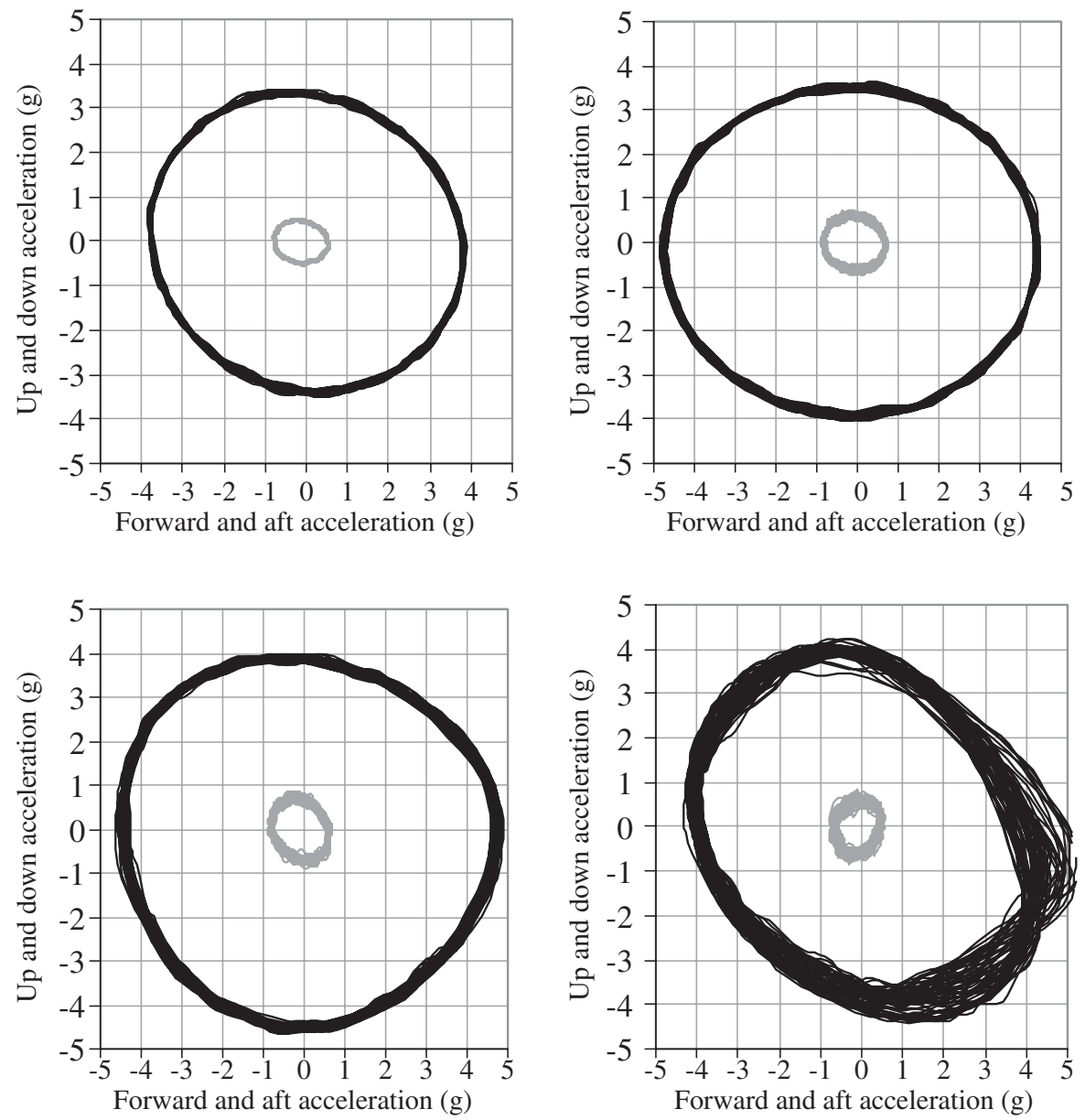

Figure 3 Drum and frame acceleration amplitudes during vibration on (clockwise from top left): rubber tires, compacted clay, PCC slab-on-grade, asphalt (up and forward directions positive).

in the vertical and horizontal directions during operation on rubber tires - consistent with linear elastic behavior. Peak-to-peak ( $p-p)$ forward/aft acceleration amplitudes exceeded $\mathrm{p}-\mathrm{p}$ vertical amplitudes by $10 \%$, creating an elliptical $x z$-diagram shape. Some difference is expected since the horizontal and vertical machine masses and inertia forces are different, and thus create variation in $p-p$ vertical and horizontal accelerations. The peak forward drum and frame accelerations occur during downward acceleration while the peak aft acceleration occurs during upward acceleration. This phase lag between $x$ and $z$ accelerations creates a rotation of the elliptical $x z$-diagram, and is likely caused by inherent yet minor nonlinearity in the compactor, e.g., drum-frame rubber isolation mounts (manufactured by Lord Corporation), mechanical linkages throughout machine.

The drum and frame $x z$-acceleration plots from operation on the compacted clay, PCC slab-on-grade, and asphalt illustrate the varying differences in response from machine vibration on rubber tires. Drum acceleration amplitudes on compacted clay, asphalt, and PCC were up to $18 \%$ greater in the downward acceleration than the upward one. Similar to rubber tires, horizontal $\mathrm{p}-\mathrm{p}$ drum acceleration was $10 \%$ greater than vertical $p-p$ on asphalt and PCC; however, horizontal $p-p$ drum acceleration was $20 \%$ greater than vertical drum acceleration on clay. The unsteady-state data exhibited on PCC stems from the nonstationary nature of testing (translation of roller) on these materials. 
Vertical and horizontal drum acceleration amplitudes are presented versus the dynamic stiffness of the underlying media in Figure 4. Using an impulse response technique (impact hammer), dynamic stiffness was determined from the $0-100 \mathrm{~Hz}$ portion of the mobility plot, where mobility is the ground surface velocity FFT spectrum divided by the force FFT spectrum [9]. The value for rubber tires could not be determined via impulse response and thus was estimated to the nearest order of magnitude $(0.1 \mathrm{kN} / \mathrm{mm})$. These dynamic stiffness values are included to simply emphasize the magnitude of variation in stiffness across the underlying materials used. Filled symbols in Figure 4 represent downward and aft acceleration amplitudes while open symbols represent upward and forward acceleration amplitudes. Despite the variability among tests on each material, Figure 4 illustrates that downward drum acceleration exceeds upward drum acceleration during machine vibration on compacted clay, asphalt, and PCC slab-on-grade. The observed trend is not consistent in the horizontal direction: aft acceleration exceeded forward acceleration during operation on compacted clay, yet forward drum acceleration exceeded aft acceleration during operation on asphalt and PCC.

Drum and frame $\mathrm{p}-\mathrm{p}$ vertical and horizontal acceleration amplitudes were normalized by $F_{\text {vib }}$ (Equation (1)) to account for variations in forcing frequency and allow direct comparison (see Figure 5). Note that vertical values are presented as open symbols and horizontal values as filled symbols. Figure 5 illustrates the increase in drum $\bar{a}_{z(\mathrm{p}-\mathrm{p})} / F_{\text {vib }}$ and $\bar{a}_{x(\mathrm{p}-\mathrm{p})} / F_{\text {vib }}$ values with underlying material stiffness. However, the subtlety of the increase suggests that acceleration amplitudes are fairly insensitive to underlying media stiffness in the frequency ranges tested.

The nonlinearity of the coupled roller compactor/underlying media system distorts the output of a sinusoidal input forcing function. The distortion is manifested in harmonic content within drum and frame vibration frequency response spectra. Frequency domain analysis of the vibration data was performed via FFT to investigate harmonic content as a measure of system nonlinearity. The FFT spectra for vibration on the four materials are presented in Figure 6, illustrating the presence of harmonic frequencies - multiples of the fundamental (applied forcing) frequency above the noise floor shown. The amplitudes of the fundamental frequency $A\left(f_{1}\right)$ and harmonics $A\left(f_{2}\right)-A\left(f_{6}\right)$ are summarized in Table 3 . The harmonic components normalized by the fundamental component $A\left(f_{i}\right) / A\left(f_{1}\right)$ and the total harmonic distortion (THD), both as percentages, are also summarized in Table 3. The THD provides a measure of collective harmonic content and is expressed
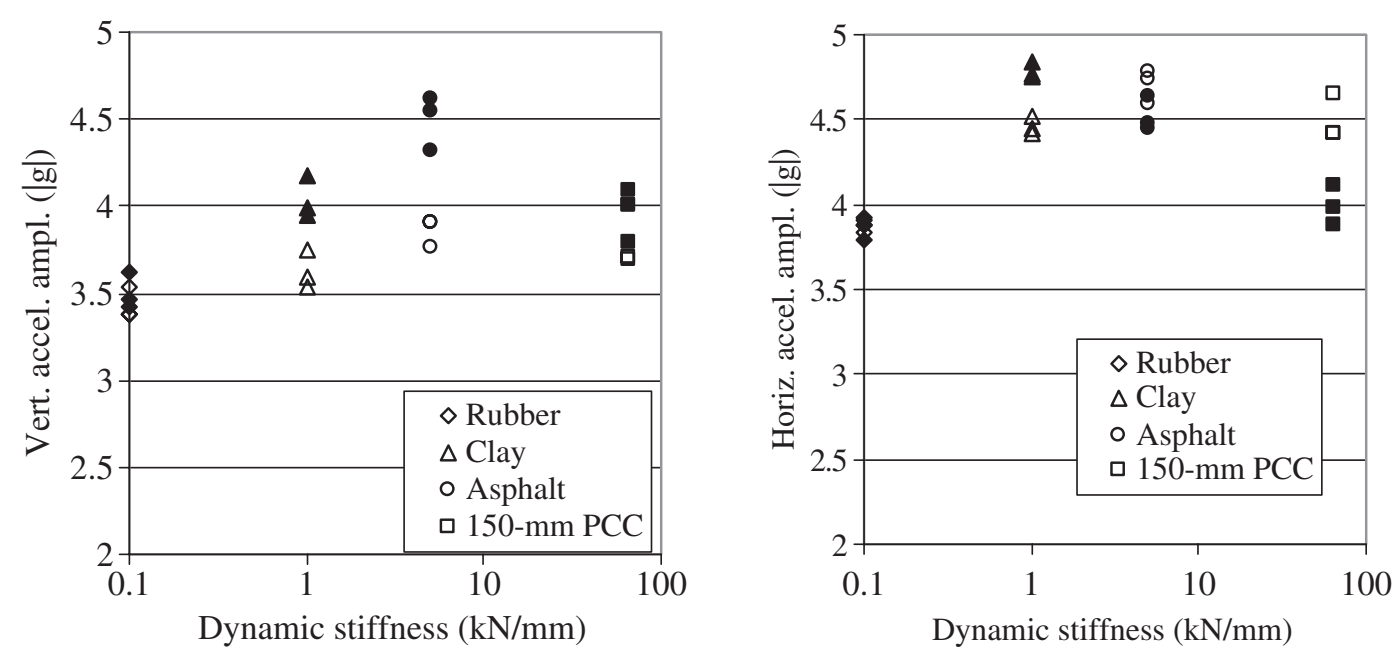

Figure 4 Peak vertical and horizontal drum accelerations $\left(f_{\text {vib }}=29 \mathrm{~Hz}\right)$. Note: filled symbols represent down and aft acceleration; open symbols represent up and forward acceleration. 

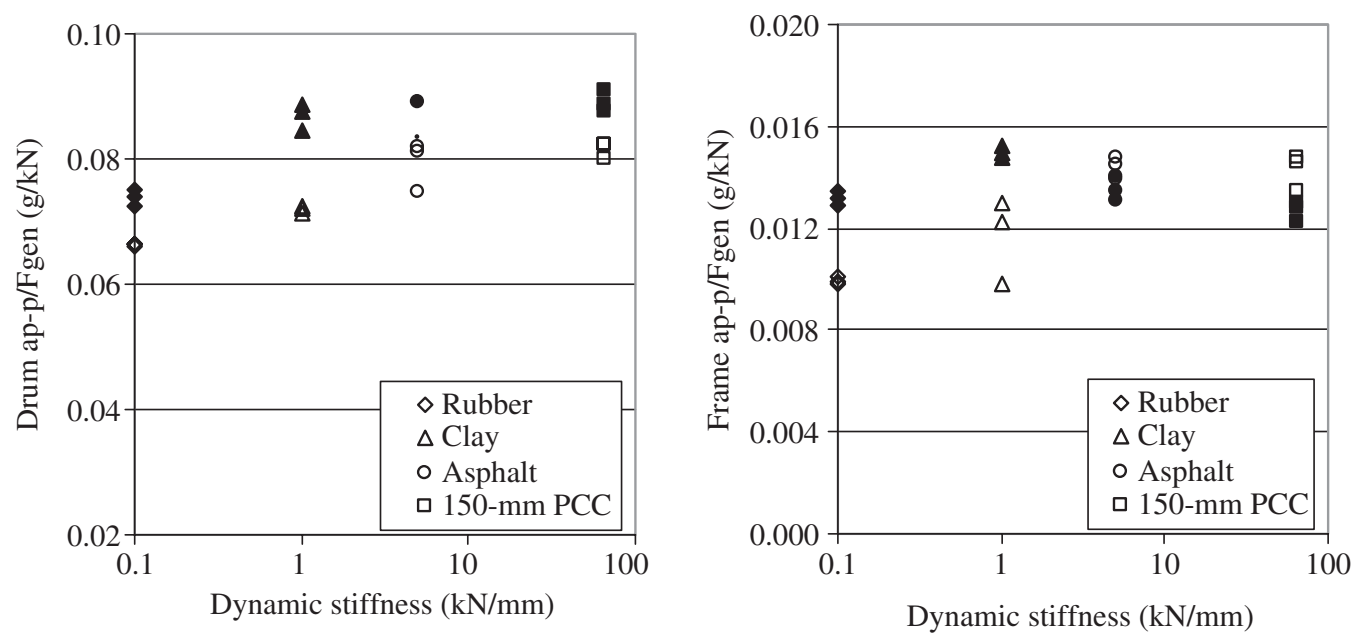

Figure 5 Normalized drum and frame acceleration amplitudes in vertical (open symbols) and horizontal (filled symbols) directions $\left(f_{\text {vib }}=29 \mathrm{~Hz}\right)$.
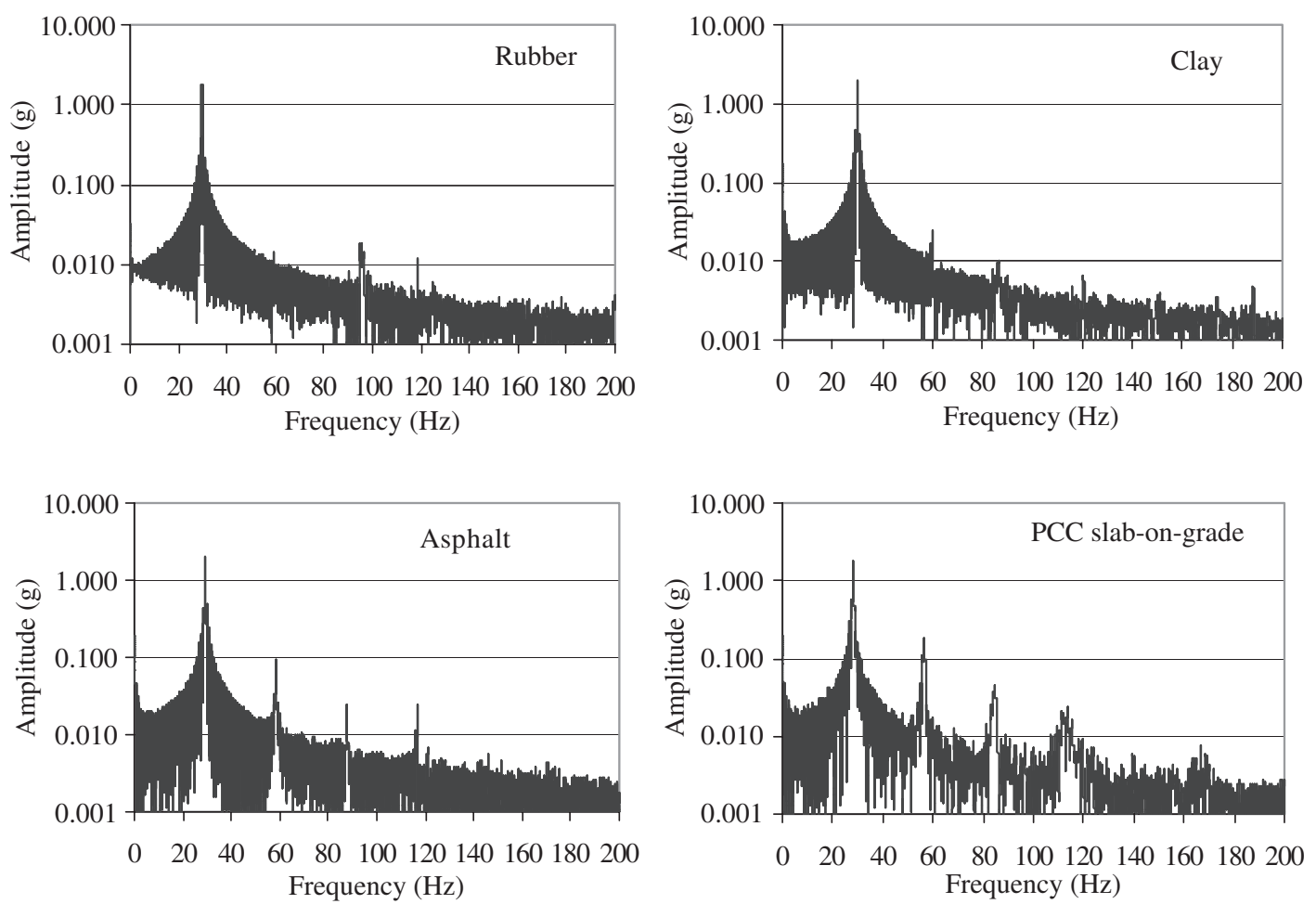

Figure 6 FFT power spectral density for vibration on nonstationary materials.

as follows:

$$
\mathrm{THD}=\frac{\sqrt{A\left(f_{2}\right)^{2}+A\left(f_{3}\right)^{2}+\cdots+A\left(f_{N}\right)^{2}}}{A\left(f_{1}\right)} \times 100
$$

As expected, the normalized frequency components and the THD were small (less than $1 \%$ ) during vibration on rubber tires. The majority of the distortion is manifested in the first harmonic $A\left(f_{2}\right)$. THD and normalized frequency components increase with underlying material 
Table 3 Frequency domain results for vibration on nonchanging material.

\begin{tabular}{|c|c|c|c|c|c|c|c|c|c|c|c|c|c|c|}
\hline \multirow[b]{2}{*}{ Test } & \multicolumn{3}{|c|}{ Rubber } & \multicolumn{3}{|c|}{ Clay } & \multicolumn{3}{|c|}{ Asphalt } & \multicolumn{3}{|c|}{ 150-mm PCC } & \multicolumn{2}{|c|}{$1-m P C C$} \\
\hline & 1 & 2 & 3 & 1 & 2 & 3 & 1 & 2 & 3 & 1 & 2 & 3 & 1 & 2 \\
\hline$f_{\mathrm{vib}}(\mathrm{Hz})$ & 29.6 & 29.0 & 29.0 & 30.1 & 29.0 & 29.2 & 29.2 & 29.7 & 29.0 & 27.6 & 27.8 & 28.2 & 22.7 & 14.9 \\
\hline $\mathrm{s}_{f}^{\mathrm{a}}(\mathrm{Hz})$ & 0.44 & 0.44 & 0.44 & 0.44 & 0.43 & 0.44 & 0.44 & 0.44 & 0.44 & 0.44 & 0.45 & 0.45 & 0.45 & 0.45 \\
\hline$A\left(f_{1}\right)(\mathrm{g})$ & 1.769 & 1.691 & 1.684 & 1.963 & 1.847 & 1.867 & 2.077 & 1.993 & 2.074 & 1.750 & 1.838 & 1.839 & 0.437 & 0.084 \\
\hline$A\left(f_{2}\right)(\mathrm{g})$ & 0.013 & 0.009 & 0.008 & 0.021 & 0.024 & 0.036 & 0.096 & 0.068 & 0.104 & 0.148 & 0.129 & 0.183 & 0.500 & 0.033 \\
\hline$A\left(f_{3}\right)(\mathrm{g})$ & 0.006 & 0.001 & 0.002 & 0.002 & 0.011 & 0.007 & 0.024 & 0.012 & 0.019 & 0.055 & 0.030 & 0.047 & 0.660 & 0.188 \\
\hline$A\left(f_{4}\right)(\mathrm{g})$ & 0.012 & 0.003 & 0.005 & 0.006 & 0.004 & 0.004 & 0.024 & 0.029 & 0.011 & 0.005 & 0.012 & 0.005 & 0.345 & 0.093 \\
\hline$A\left(f_{5}\right)(\mathrm{g})$ & 0.003 & 0.002 & 0.001 & 0.003 & 0.003 & 0.003 & 0.005 & 0.004 & 0.004 & 0.006 & 0.006 & 0.003 & 0.199 & 0.026 \\
\hline$A\left(f_{6}\right)(\mathrm{g})$ & 0.004 & 0.002 & 0.002 & 0.002 & 0.005 & 0.004 & 0.002 & 0.001 & 0.003 & 0.002 & 0.002 & 0.003 & 0.093 & 0.020 \\
\hline THD $(\%)$ & 1.12 & 0.58 & 0.61 & 1.15 & 1.46 & 2.01 & 4.90 & 3.77 & 5.12 & 9.02 & 7.27 & 10.29 & 211 & 255 \\
\hline$A\left(f_{2}\right) / A\left(f_{1}\right)(\%)$ & 0.75 & 0.54 & 0.50 & 1.08 & 1.28 & 1.95 & 4.61 & 3.41 & 5.00 & 8.45 & 7.04 & 9.97 & 114.4 & 39.1 \\
\hline$A\left(f_{3}\right) / A\left(f_{1}\right)(\%)$ & 0.36 & 0.05 & 0.13 & 0.11 & 0.59 & 0.37 & 1.18 & 0.61 & 0.94 & 3.12 & 1.62 & 2.54 & 151.1 & 222.8 \\
\hline$A\left(f_{4}\right) / A\left(f_{1}\right)(\%)$ & 0.69 & 0.16 & 0.30 & 0.32 & 0.22 & 0.21 & 1.15 & 1.47 & 0.51 & 0.27 & 0.65 & 0.26 & 78.9 & 110.8 \\
\hline$A\left(f_{5}\right) / A\left(f_{1}\right)(\%)$ & 0.17 & 0.10 & 0.06 & 0.15 & 0.15 & 0.17 & 0.25 & 0.19 & 0.19 & 0.33 & 0.34 & 0.18 & 45.6 & 31.2 \\
\hline$A\left(f_{6}\right) / A\left(f_{1}\right)(\%)$ & 0.22 & 0.13 & 0.11 & 0.08 & 0.27 & 0.20 & 0.09 & 0.06 & 0.14 & 0.11 & 0.09 & 0.14 & 21.2 & 24.2 \\
\hline
\end{tabular}

alHalf-power bandwidth.

stiffness (see Figure 7). Note that the decoupling during vibration on 1-m PCC yields significant nonlinearity and thus harmonic content. Table 3 and Figure 7 illustrate that the percentage of harmonic content in the drum acceleration is more sensitive to underlying material stiffness than drum acceleration amplitude. While normalized drum acceleration amplitude increased 24\% from operation on rubber to operation on 150 -mm PCC, the THD increased over $1000 \%$ from operation on rubber to operation on 150-mm PCC.

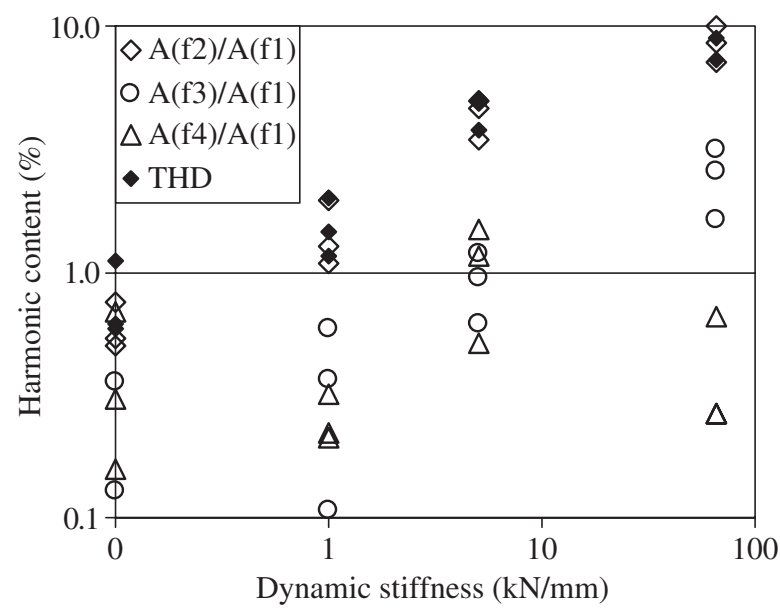

Figure 7 Normalized harmonic content and total harmonic distortion as percentage during vibration on nonstationary materials.

\section{Phase Two Test Results: Vibration During Soil Compaction}

The instrumented vibratory roller was driven over the prepared $25 \mathrm{~m}$ long sand bed at a constant velocity of $0.5 \mathrm{~m} / \mathrm{s}$. Drum and frame acceleration amplitudes during a typical pass are shown in Figure 8. Four locations labeled A, B, $\mathrm{C}$, and D in Figure 8 and measured at 20, 40, 60, and $80 \%$ along the bed length, were marked for independent testing of soil properties. A nuclear gage was used to determine soil density and moisture content. A dynamic cone penetrometer (DCP) was used to measure penetration resistance, a surrogate of soil stiffness and shear strength (see Figure 8). Three successful compaction tests were performed on sand, denoted test beds S2, S3, and S4. Data acquisition problems prevented the analysis of S1 data. Each sand bed was prepared by tilling a $300-\mathrm{mm}$ thick soil lift to a homogeneous state. It should be noted that the sand beds rested atop a $1.5 \mathrm{~m}$ fill embankment of similar sand. Once the soil was tilled to a homogeneous loose state with the desired water content, a single pass of the compactor was performed. Density, moisture content, and DCP testing were then conducted at points $\mathrm{A}, \mathrm{B}, \mathrm{C}$, and D. Pass two of the roller compactor followed. This process of rolling and testing was repeated until the desired density was achieved (typically $5-8$ passes). 


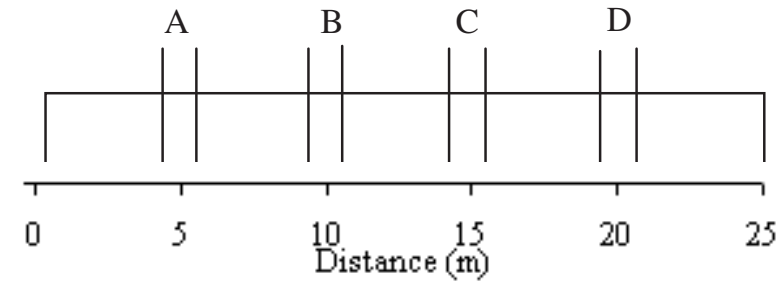

(a)

(d)

(b)
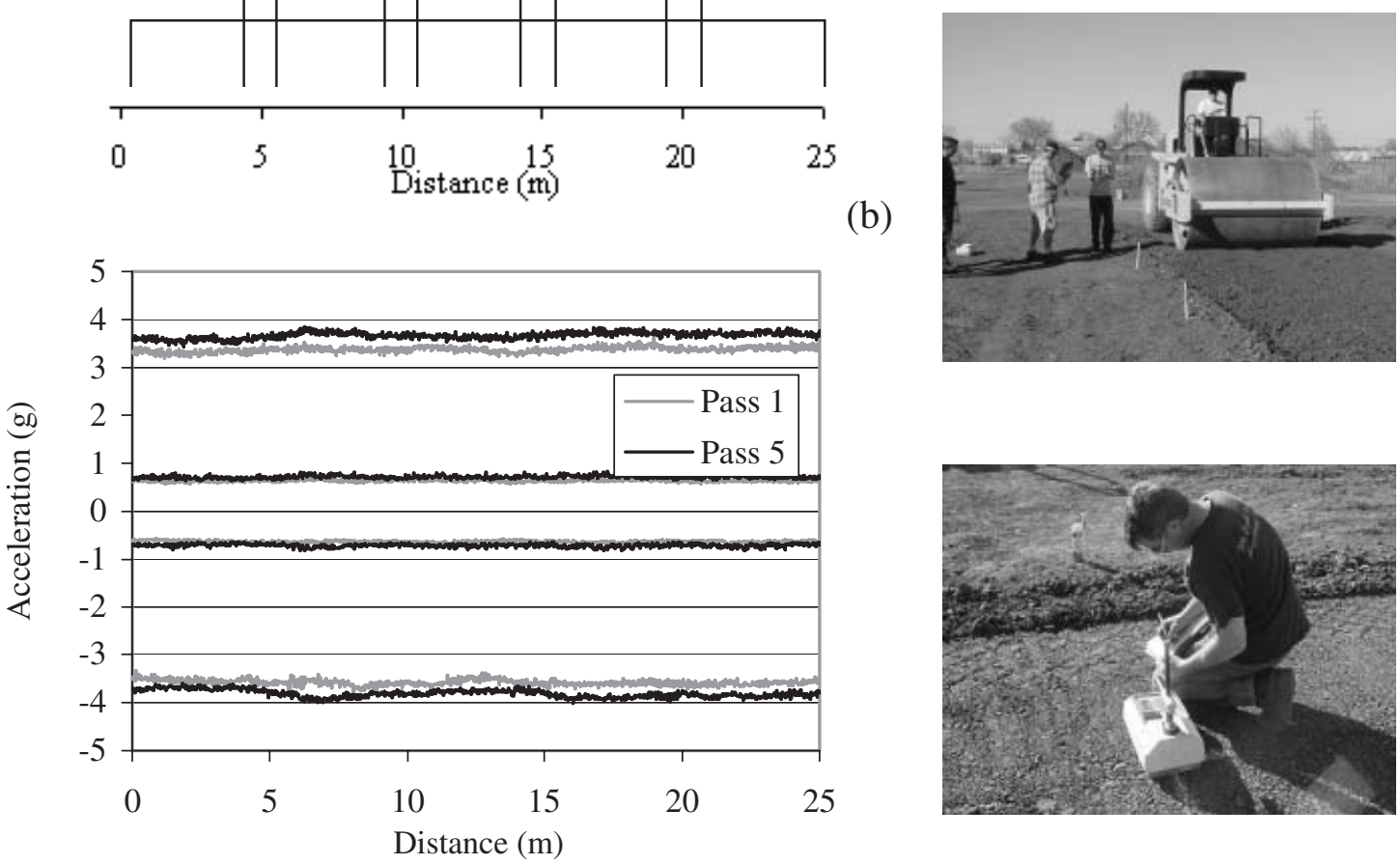

(c)
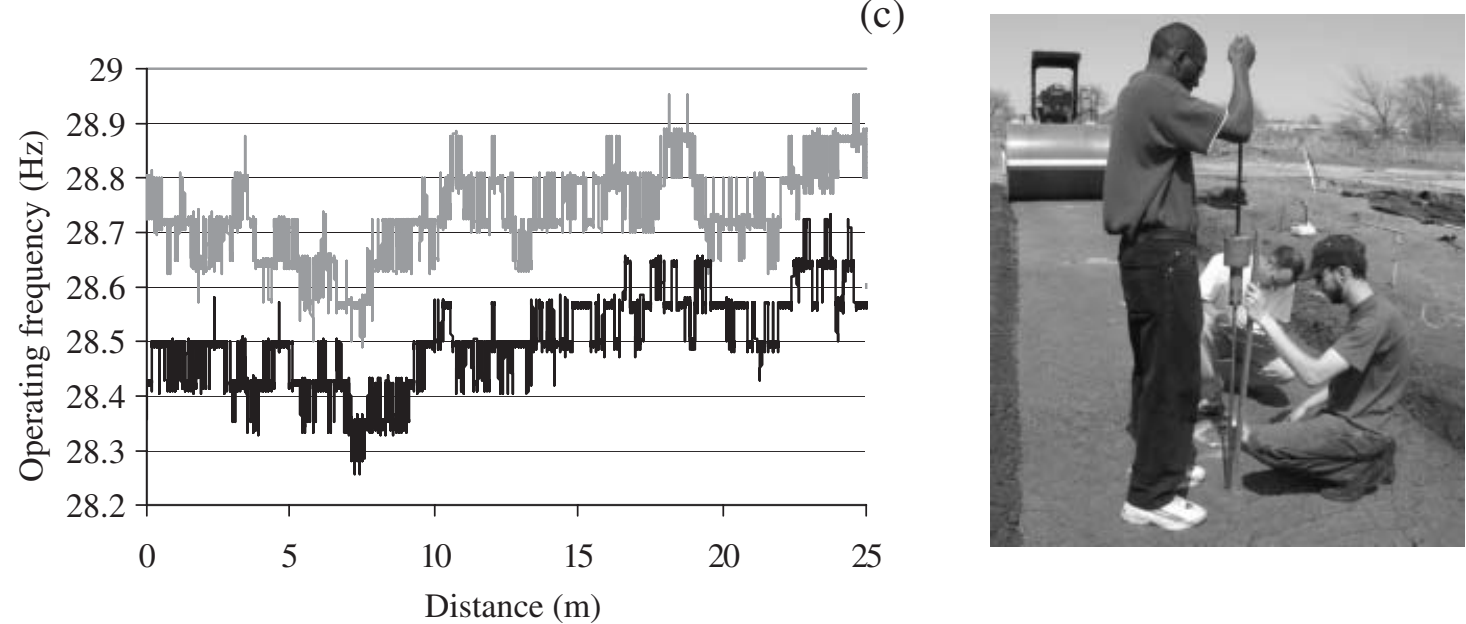

Figure 8 Overview of testing procedure: (a) locations of positions A-D; (b) time history of drum and frame acceleration amplitudes during testing; (c) variation in vibration frequency during operation; (d) acquiring data during a pass, performing a nuclear gage test, and performing a dynamic cone penetration test.

Peak vertical drum accelerations measured at locations A-D during compaction of sand beds S2, S3, and S4 are presented in Figure 9 versus percent compaction. Percent compaction is defined as the percentage ratio of dry density (dry soil mass/total volume) to a standard Proctor maximum dry density for that particular soil as determined via laboratory testing (ASTM D698). Design and construction requirements typically specify that a contractor must achieve percent compaction of $95-100 \%$ to provide adequate soil stiffness and strength. Percent compaction values of $97-101 \%$ were achieved during compaction of test beds S2, S3, and S4. During 


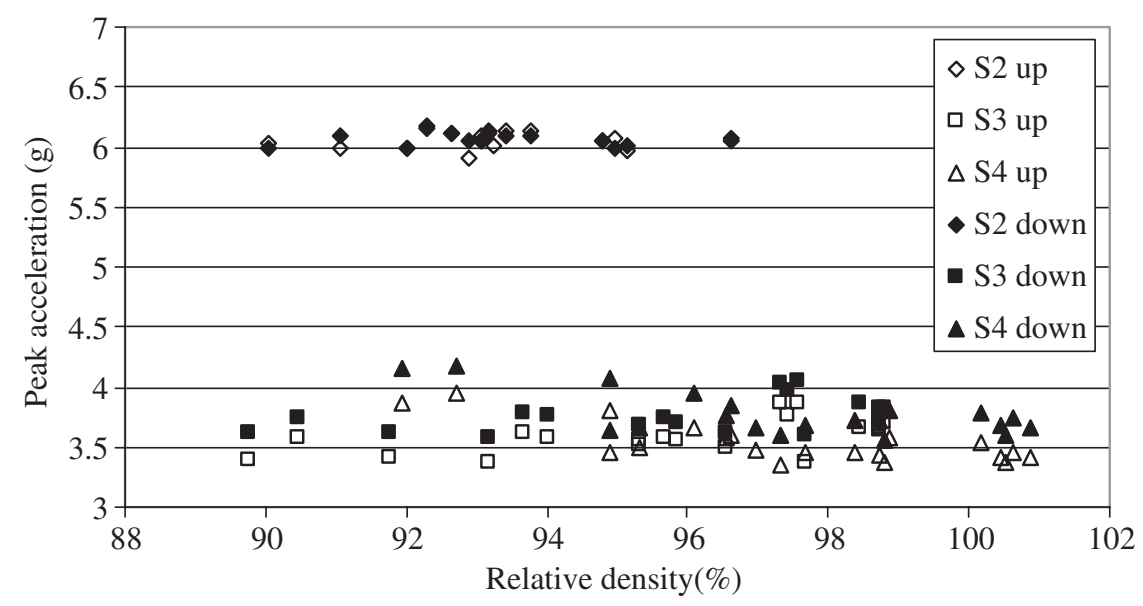

Figure 9 Peak drum accelerations during compaction of sand (vibration frequency for each test: $29 \mathrm{~Hz}$ for S3 and S4; $38 \mathrm{~Hz}$ for S2).

vibratory compaction, significant compaction is achieved during the first pass. Despite beginning in a tilled loose state, test beds $\mathrm{S} 2-\mathrm{S} 4$ reached $90-92 \%$ compaction after the first pass. Therefore, vibration features must be sensitive enough to changes during compaction from $90 \%$ compaction to $95-100 \%$ compaction. Though this percent compaction increase is small $(5-8 \%)$, during laboratory testing, soil modulus increased $50-75 \%$ during compaction from $90-95 \%$ compaction [10].

The data presented in Figure 9 represents average upward (open symbols) and downward (filled symbols) drum acceleration amplitudes measured as the compactor passed over points A-D during five successive passes of the roller compactor. Each data point is the average of $2 \mathrm{~s}$ worth of data taken during drum passage over $1 \mathrm{~m}$ of test bed (see points A-D in Figure 8). The acceleration levels measured at any time during compaction reflect machine vibration upon a complex body of 'involved' soil (i.e., the soil that is stressed by the vibrating drum at a given time and location). The involved soil ahead of the drum is less dense than the involved soil behind the drum. Hence, the drum and frame vibration characteristics during any pass reflect machine vibration on soil that varies from prepass density ahead of the roller to post-pass density behind the drum. It should be noted that associated density and DPI values were measured after each pass.
Figure 9 illustrates the effect of vibration frequency on drum acceleration. Test beds S3 and S4 were compacted at $f_{\text {vib }}=29 \mathrm{~Hz}$ while test $\mathrm{S} 2$ was performed at $f_{\mathrm{vib}}=38 \mathrm{~Hz}$; hence the increased drum accelerations for test S2. Figure 9 shows that the downward drum acceleration exceeds the upward drum acceleration by $10-15 \%$ throughout compaction of beds $\mathrm{S} 3$ and $\mathrm{S} 4$, yet remains symmetric at the $38 \mathrm{~Hz}$ compaction of bed $\mathrm{S} 2$. Therefore, the difference between upward and downward drum acceleration does not appear to be sensitive to the changes in soil properties during sand compaction.

Drum vibration amplitudes exhibited little change during compaction of beds S2, S3, and S4 (Figure 9); however, when normalized by $F_{\mathrm{vib}}$, a slight increase is observed as soil densifies and stiffens (see Figure 10). A significant hurdle for vibration-based condition monitoring of soil compaction is the inherent operational variability due to shifting vibration frequency. The Ingersoll-Rand SD-100D employs open-loop manual frequency control (lever-controlled). Further, hydraulic power is shared by the drive motor, steering motor, and vibration motor. For safety reasons, steering and drive receive highest priority. As a result, vibration frequency varies during operation (see Figure 8) and tends to decrease as the underlying soil stiffens. For example, $f_{\text {vib }}$ varied by $\pm 2 \mathrm{~Hz}$, during compaction of beds S3 and S4. Figure 10 shows that the normalized peak to peak acceleration generally 


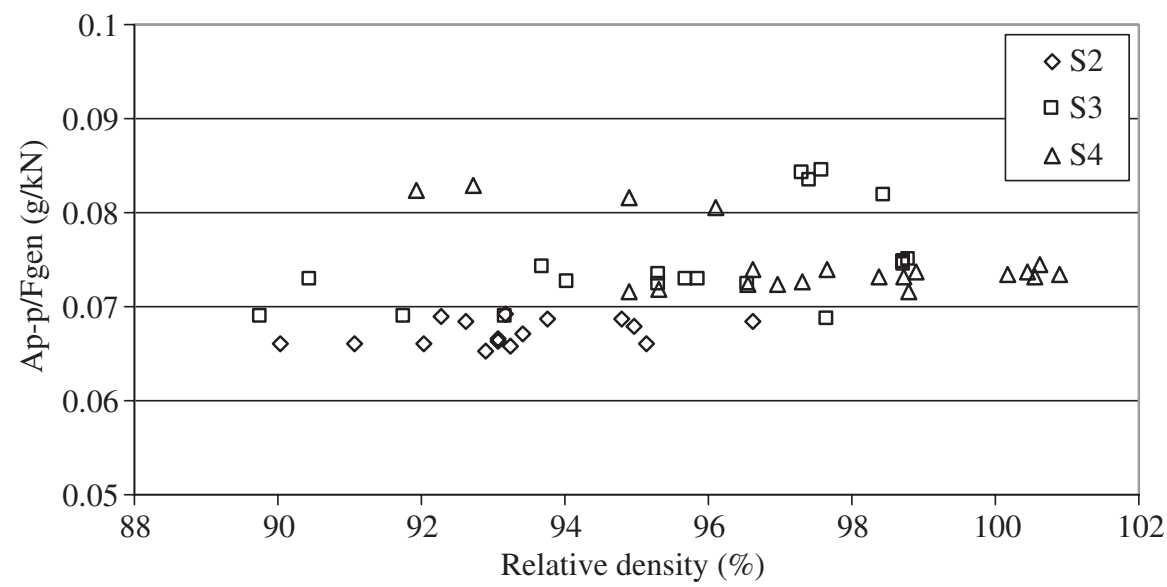

Figure 10 Normalized peak-to-peak accelerations during compaction of sand.

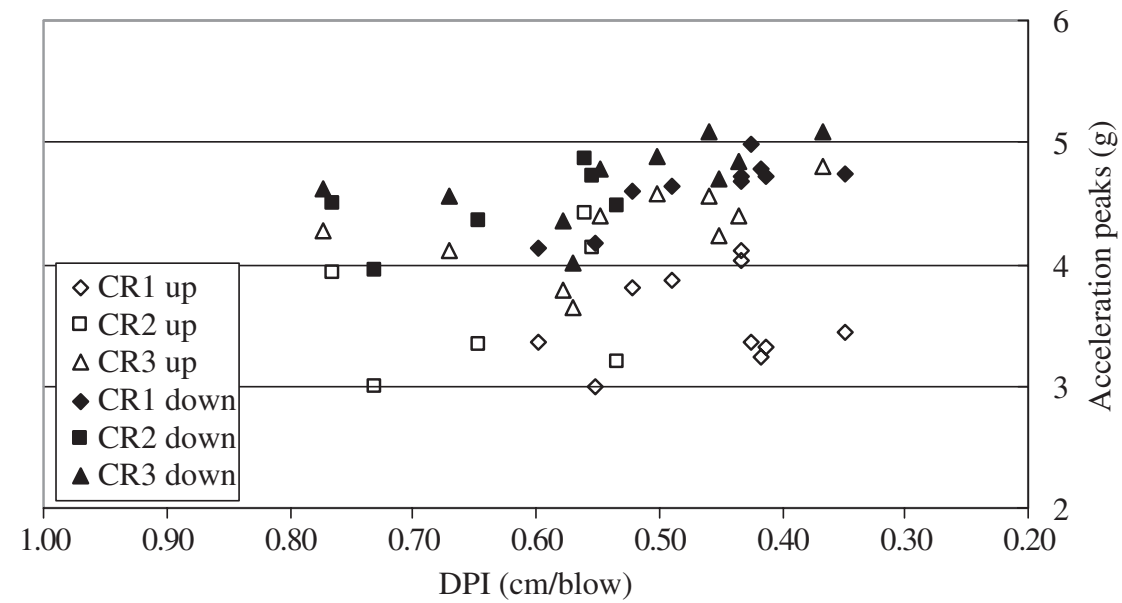

Figure 11 Peak accelerations during compaction of crushed rock (average vibration frequency for each test: $20-26 \mathrm{~Hz}$ for $\mathrm{CR} 1$ and $\mathrm{CR} 2 ; 26-28 \mathrm{~Hz}$ for CR3).

increases during soil compaction. While it is somewhat intuitive that drum acceleration increases as the underlying material stiffness increases, the sensitivity of drum acceleration amplitude as a feature for health monitoring appears limited for the frequencies investigated.

Compaction was also performed on three crushed rock test beds labeled CR1, CR2, and CR3. Due to site restrictions, the test bed length was limited to $10 \mathrm{~m}$. The thickness of test beds $\mathrm{CR} 1$ and CR2 were tapered; location A $(2.5 \mathrm{~m}$ along test bed) and location $\mathrm{B}(7.5 \mathrm{~m}$ along test bed) were 300 and $175 \mathrm{~mm}$ thick, respectively. The thickness of test bed CR3 was $300 \mathrm{~mm}$ along the entire length. The compacted subsurface beneath the CR lifts had served as a staging area for dump trucks, graders, and loaders; hence, it was extremely stiff. Excitation frequency varied from $20-26 \mathrm{~Hz}$ during testing of CR1 and CR2 and from $26-28 \mathrm{~Hz}$ during testing of CR3. Vertical drum acceleration amplitudes observed during compaction of crushed rock are presented in Figure 11. The amplitudes are plotted versus dynamic penetration index (DPI), measured in centimeters of penetration per hammer blow. DPI is an effective measure of soil stiffness and is inversely proportional to stiffness [11]. Figure 11 reveals a general trend wherein the drum acceleration increases as the DPI decreases (and the soil stiffens). During compaction of crushed rock, 
the downward drum acceleration was much greater (up to $50 \%$ ) than the upward drum acceleration. The vibration frequency also varied considerably within passes of the compactor and from pass to pass. Normalized drum accelerations $\bar{a}_{z(\mathrm{p}-\mathrm{p})} / F_{\text {vib }}$ are presented in Figure 12. As was the case during compaction of sand, normalized acceleration amplitudes generally increase as the crushed rock layers densified and stiffened; however, the increase is slight.

Frequency response (FFT) analysis was performed on the two-second data files recorded at points A-D during each compaction pass over test beds S2-S4 and CR1-CR3. The vertical drum vibration THD was calculated as per Equation (1) and is presented in Figures 13 and
14 for the sand and crushed rock test beds. Values of $A\left(f_{3}\right)$ through $A\left(f_{N}\right)$ were insignificant compared to $A\left(f_{2}\right)$; hence, the THD essentially reflects the ratio $A\left(f_{2}\right) / A\left(f_{1}\right)$. Figure 14 illustrates THD values from 1 to $5 \%$ during operation on the sand beds and $8-20 \%$ during operation on the crushed rock beds. There is considerable variability in THD from test to test. Figure 14 illustrates an increasing trend in THD as soil stiffens. The increase in THD during vibration on the sand beds is subtle; the increase in THD during compaction of crushed rock is substantial.

While the behavioral trends during vibratory compaction are similar on both soils, i.e., increasing normalized vibration amplitudes and THD

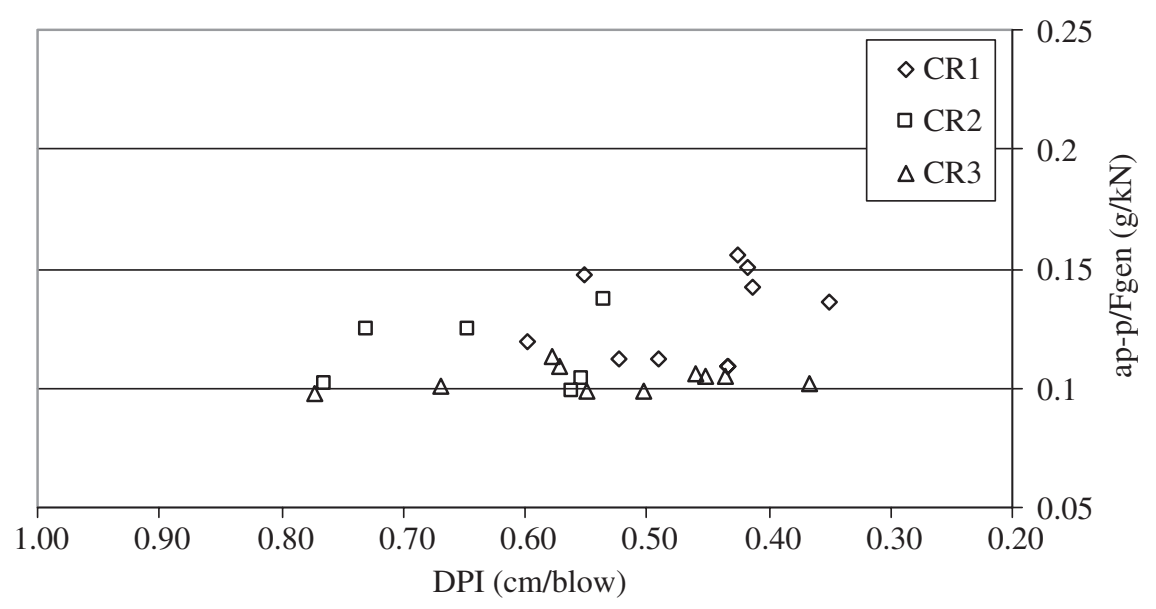

Figure 12 Normalized peak-to-peak accelerations during compaction of crushed rock.

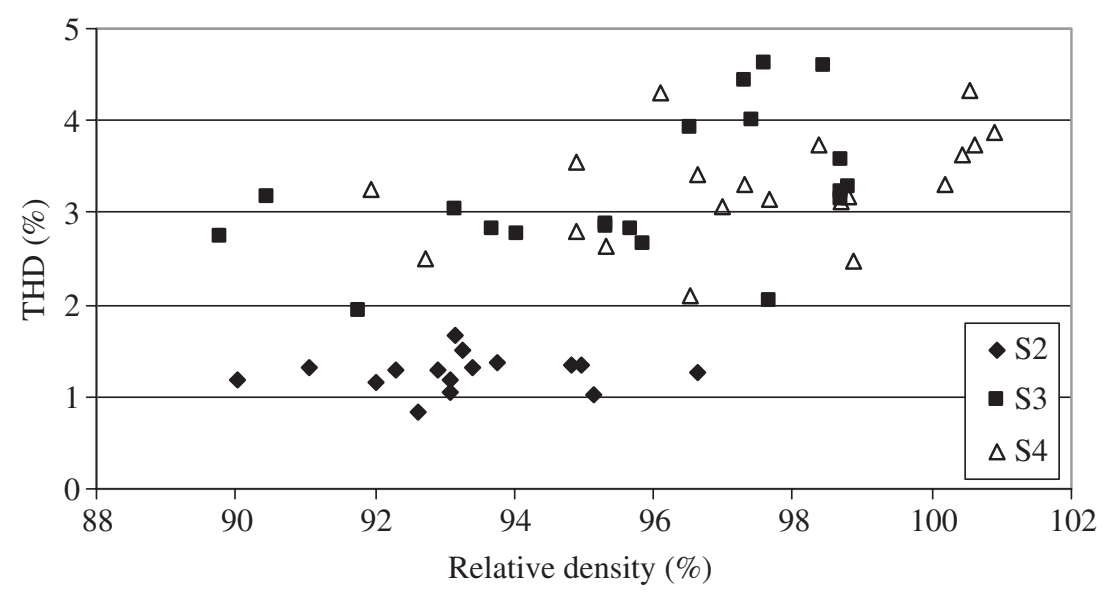

Figure 13 Total harmonic distortion during compaction of sand. 


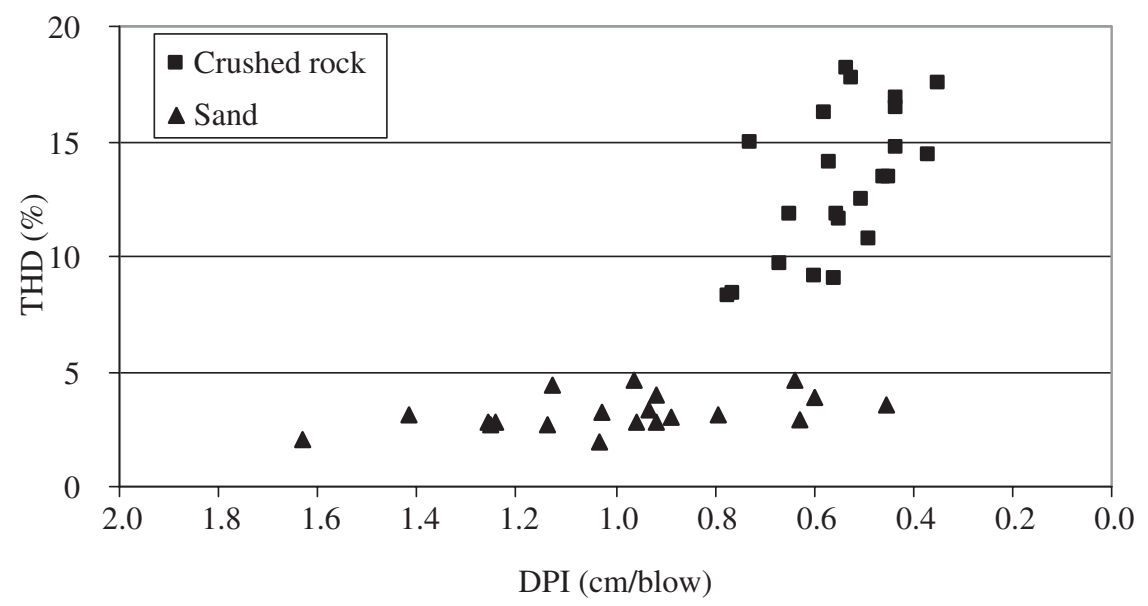

Figure 14 Total harmonic distortion during compaction of sand and crushed rock.

values with increasing underlying stiffness, the data revealed considerable differences in the magnitude of vibration amplitude and THD between the sand and crushed rock sites. Normalized drum acceleration amplitudes were twice as large during operation on the crushed rock site than during vibration on the sand site. The difference in upward versus downward drum acceleration was also much greater during compaction of crushed rock. THD measured during vibration on the crushed rock site were more than twice those measured during operation on the sand site. These differences can be attributed to the effect of both material properties and underlying strata. The soil 'involved' in the coupled roller compactor/soil system extends below the $150-300 \mathrm{~mm}$ lift of soil undergoing compaction; hence, the underlying strata can influence the observed roller vibration characteristics. As illustrated by the DPI profiles collected after compaction of both sand and crushed rock sites (see Figure 15), the crushed rock and underlying embankment is stiffer than the sand site stratigraphy. It is also possible that the drum uncoupled from the ground during vibration upon crushed rock

If proven effective, VBHM techniques could be used to estimate the condition of the geostructure, e.g., percent compaction and stiffness of underlying soil. This inverse problem would require a relationship between measured vibration features (e.g., THD, normalized drum amplitude, or changes therein) and the sought after earth structure properties. In a nonphysical modelbased approach, a statistical relationship could be developed. Alternatively, a physical model of the coupled system, for example lumped parameter model of the compactor coupled with lumped parameter or continuum model of the involved soil, could be developed. In either case, the input to the model structure would be the measured vibration features; the output would be the desired soil properties.

While the data presented here shows promise for VBHM of earth structures, continued testing, modeling, and data analysis is required to identify robust vibration features. For example, drum acceleration and THD values depend upon the near surface soil of interest and the underlying stratigraphy. The underlying stratigraphy varies considerably from site to site and even within a site, e.g., along miles of highway subgrade. Ideally, VBHM of earth structures could effectively be used on a site with no prior information. This will likely require multiple sensors and multilayered models of the earth structure so that the response of the layer of interest can be isolated. However, VBHM may require some degree of a priori information about the subsurface, e.g., periodic calibration at each site. In the case of compaction, a priori information could also be provided by georeferencing to previous data through repeated passes over the same area. 


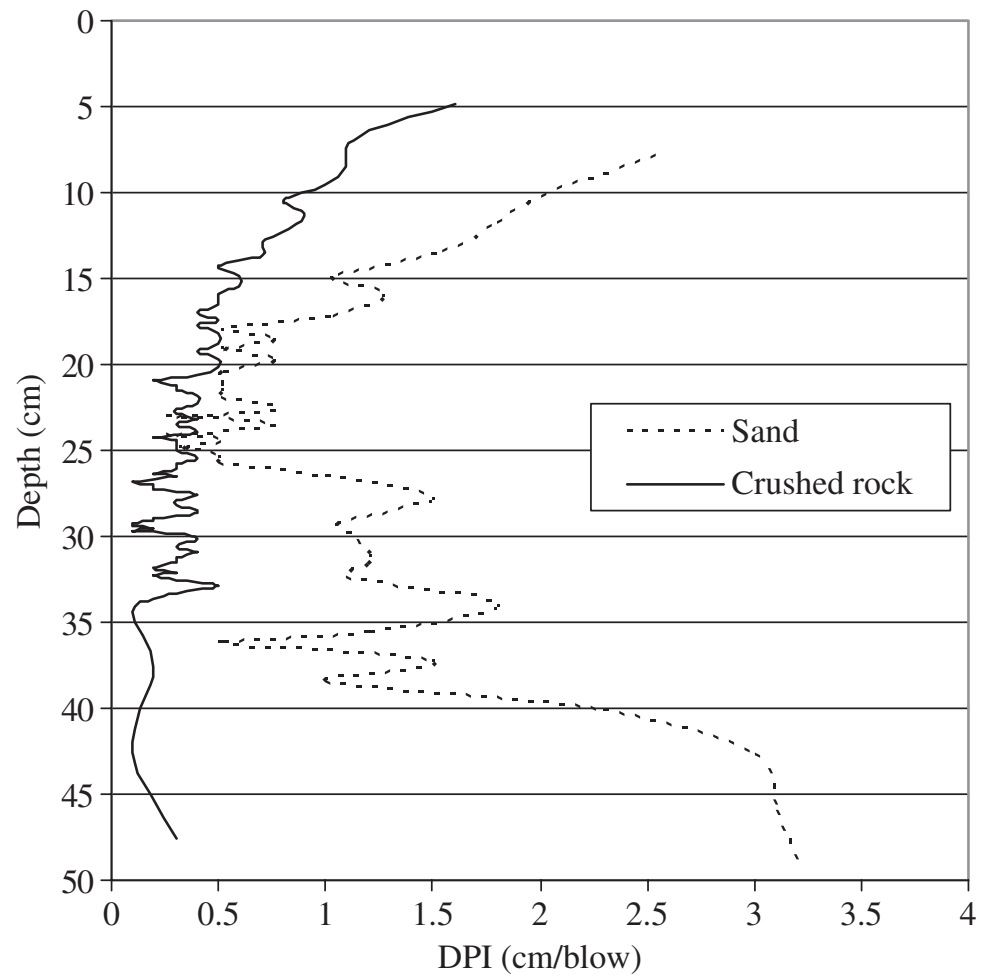

Figure 15 Dynamic cone penetration profiles of sand and crushed rock test beds and underlying strata.

\section{Summary and Conclusions}

An experimental program was carried out to explore the efficacy of VBHM of earth structures. The drum and frame of an Ingersoll-Rand compactor were instrumented with accelerometers. Vibration tests were carried out on various nonchanging materials to determine the sensitivity of various time- and frequency-domain features to changes in underlying material stiffness. Vibratory compaction was then carried out on carefully prepared beds of sand and crushed rock to explore vibration characteristics and changes therein as the soil stiffens during compaction. The testing program provided the following findings:

1. At the frequencies tested, time-domain drum and frame acceleration amplitudes were fairly insensitive to changes in underlying material properties. During testing on non-changing media, though the underlying material stiffness increased by orders of magnitude, normalized acceleration amplitudes increased less than $25 \%$. During soil compaction, normalized drum acceleration values increased slightly (less than 10\%) as the soil stiffness increased more than twice.

2. Harmonic content, measured during vibration and expressed as normalized frequency components and THD, exhibited much greater sensitivity to changes in underlying material properties. THD increased over $1000 \%$ from operation on rubber to operation on $150-\mathrm{mm}$ PCC. THD increased $50-100 \%$ during compaction of sand and crushed rock.

3. Magnitudes of measured roller vibration characteristics, normalized drum acceleration, and THD, differed considerably at the sand site and crushed rock site due to both material effects and the effect of underlying strata. Given the existing variability in earthwork stratigraphies, vibration and THD amplitudes alone would not be sufficient features.

4. Operational variability issues present a challenge to VBHM. Fluctuations in vibration 
frequency, forward velocity, local variability in soil moisture and composition, and depth and stiffness of underlying strata all effect vibration characteristics. Machine variability can be addressed via control technology; however, the analysis techniques will have to be further developed to accommodate inherent soil variability.

These test results provide some promise for VBHM of earth structures; the compactor's vibration characteristics are influenced by the underlying material. However, the testing herein was limited to vibration on two soil sites with only one amplitude setting and limited vibration frequencies. Further testing is needed to explore vibration response and sensitivity to underlying media over a broad band of frequencies, e.g., $10-50 \mathrm{~Hz}$, and multiple amplitudes. Further, to better characterize the relative effects of both the surface soil and the underlying media on vibration response, controlled testing of various soil types overlying the same subsurface stratigraphy, and of the same surface soil resting upon various underlying stratigraphies, is required. This data would then enable the development of robust model structures that would predict the soughtafter soil properties, e.g., stiffness, based upon the measured vibration characteristics.

\section{Acknowledgments}

The authors are very grateful to the Oklahoma Department of Transportation and the National Science Foundation (CMS-0327509) for providing funding for this research program. The authors would also like to express their appreciation to the Ingersoll-Rand Corporation for providing the vibratory roller compactor used in this study.

\section{References}

1. Geistlinger, L. (1996). Onboard compaction meters make inroads into U.S. market. Roads and Bridges, (August), 40-42.

2. Thurner, H.F. (2001). Quality assurance and self control in road construction advanced measurement technology. In: Proc. 4th Int. Symp. Infrastructure Construction Systems and Technologies, pp. 72-81.

3. Farrar, C.R., Doebling, S.W. and Nix, D.A. (2001). Vibration-based structural damage identification. Phil. Trans. R. Soc. Lond. A, 359, 131-149.

4. Sohn, H., Allen, D.W., Warden, K. and Farrar, C.R. (2003). Statistical damage classification using sequential probability ratio tests. Structural Health Monitoring, 2(1), 57-75.

5. Doebling, S.W. and Farrar, C.R. (2001). Estimation of statistical distributions for modal parameters identified from averaged frequency response function data. J. Vibration and Control, 7, 603-624.

6. Sohn, H. and Law, K.H. (2001). Extraction of ritz vectors from vibration test data. Mechanical Systems and Signal Processing, 15(1), 213-226.

7. Mitchell, J.S. (1992). Introduction to Machinery Analysis and Monitoring, Tulsa, OK: PenWel Books.

8. Mooney, M.A., Gorman, P.B., Farouk, E., Gonzalez, J.N. and Akanda, A.S. (2003). Exploring vibrationbased intelligent soil compaction. Oklahoma Department of Transportation. Project No. 2146 Final Report, 250 pp.

9. Davis, A., Hertlein, B., Lim, M. and Michols, K. (1996). Impact-echo and impulse response stress wave methods. In: Proc. Nondestructive Evaluation of Bridges and Highways, 4-5 Dec., Scottsdale, Az. pp. 88-96.

10. Akanda, A.S. (1999). Effect of moisture content \& clay fraction on the cyclic behavior of soil during compaction. MS Thesis, University of Oklahoma. 98 pp.

11. Rahim, A.M. and George, K.P. (2002). Automated dynamic cone penetrometer for subgrade resilient modulus characterization. Transportation Research Record, No. 1806, pp. 70-77. 